\title{
THE SYNTHESIS, REACTIVITY AND THE ANTIMICROBIAL ACTIVITY OF SUBSTITUTED THIENO[2,3-d]PYRIMIDINE- 4(3H)-THIO(SELENO)NES
}

\author{
D.O.Kolomieitsev ${ }^{1}$, V.I.Markov ${ }^{1}$, V.O.Astakhina ${ }^{1}$, S.I.Kovalenko ${ }^{2}$, S.A.Varenichenko ${ }^{1}$, \\ O.V.Kharchenko ${ }^{1}$ \\ ${ }^{1}$ Ukrainian State University of Chemical Technology \\ 8, av. Gagarina, Dnepropetrovsk, 49005, Ukraine. E-mail: dimakolomeicev@gmail.com \\ ${ }^{2}$ Zaporozhye State Medical University
}

Key words: thieno[2,3-d]pyrimidine-4(3H)-thio(seleno)nes; alkylation; antibacterial activity

\begin{abstract}
A new series of R1,R2-thieno[2,3-d]pyrimidine-4(3H)-one, thione and selenone derivatives have been synthesized; the reaction of alkylation of the compounds obtained has been studied. Their structures have been confirmed by the NMR ${ }^{1} \mathrm{H}$ and mass spectra, and elemental analysis. The antibacterial and antifungal activities in vitro against three bacterial and two fungal pathogens have been revealed using the stiff plate agar diffusion method and the serial dilution method. The minimal bactericidal, fungicidal and bacteriostatic concentrations have been obtained. The pharmacological screening has shown that some of the compounds obtained possess a good antimicrobial activity. The culture of S.aureus. appeared to be the most sensitive to compound 10a. The best fungistatic indicators against A.niger have been found for compounds $4 a$ and 9 a.
\end{abstract}

\begin{abstract}
СИНТЕЗ, РЕАКЦІЙНА ЗДАТНІСТЬ ТА АНТИМІКРОБНА АКТИВНІСТЬ ЗАМІЩЕНИХ ТІЄНО[2,3-d]ПІРИМІДИН4(3Н)-TIO(СЕЛЕНО)НІВ

Д.О.Коломійцев, В.І.Марков, В.О.Астахіна, С.І.Коваленко, С.А.Варениченко, О.В.Харченко

Ключові слова: тієно[2,3-d]піримідин-4(3Н)-тіо(селено)ни; алкілування; антибактеріальна активність Синтезовано ряд нових похідних R1,R2-тієно[2,3-d]піримідин-4(3H)-онів, тіонів і селенонів та вивчено реакцію алкілування отриманих продуктів. Структури отриманих продуктів встановлені за допомогою даних ЯМР ${ }^{1} \mathrm{H}$ та мас-спектрів і даних елементного аналізу. Антибактеріальну і фунгістатичну активність in vitro проти 3 культур бактерій і двох видів грибів було встановлено за допомогою методу дифузії речовини в агар і методу серійних розведень. Були визначені мінімальні концентрації бактеріальної, фуунгістатичної і бактеріостатичної активності. Фармакологічне дослідження підтвердило, що деякі з отриманих продуктів проявляють добру антимікробну активність. Культура бактерії S. aureus. найбільш чутлива до сполуки 10а. Найкращі фунгістатичні показники проти цвільового гриба A.niger виявлені для сполук 4а і 9а.
\end{abstract}

СИНТЕЗ, РЕАКЦИОННАЯ СПОСОБНОСТЬ И АНТИМИКРОБНАЯ АКТИВНОСТЬ ЗАМЕЩЕННЫХ ТИЕНО[2,3-С] ПИРИМИДИН-4(ЗН)-ТИО(СЕЛЕНО)НОВ

Д.О.Коломейцев, В.И.Марков, В.О.Астахина, С.И.Коваленко, С.А.Варениченко, А.В.Харченко

Ключевые слова: тиено[2,3-d]пиримидин-4(3Н)-тио(селено)ны; алкилирование; антибактериальная активность

Синтезирован ряд новых производных R1,R2-тиено[2,3-d]пиримидин-4(3H)-онов, тионов и селенонов, изучена реакция алкилирования полученных соединений. Структуры полученных соединений установлены с помощью данных ЯМР ${ }^{1} \mathrm{H}$ и масс-спектров, а также данных элементного анализа. Антибактериальная и фунаистатическая активность in vitro против трёх видов бактерий и двух видов грибов была обнаружена с помощью метода дифрфузии вещества в агар и метода серийного разведения. Определены минимальные концентрации бактериальной, фунгицидной и бактериостатической активности. Фармакологическое исследование подтвердило, что некоторые из полученных продуктов проявляют хорошую антимикробную активность. Культура бактерии S.aureus наиболее чувствительна к соединению 10 . Наилучшие фунгистатические показатели против цвелевого гриба A.niger определены для соединений 4 а и 9 a.

Thieno $[2,3-d]$ pyrimidines are of great interest in pharmaceutical industry due to the potent physiological properties such as blood platelet aggregation inhibition, the antiviral and anti-inflammatory activity [1-3]. Molecular docking also shows that thieno[2,3-d] pyrimidines reveal the increased activity as inhibitors for PARP-1 (poly(ADP-ribose)-polymerase-1) [4]. Although the synthesis of thieno[2,3-d]pyrimidines has attracted much attention in organic synthesis, so far only few methods have been reported for the synthesis of benzothieno[2,3- $d$ ] pyrimidines [5, 6]. Moreover, thieno[2,3-d]pyrimidines containing active functional groups are especially of great interest. The presence of different groups $(\mathrm{R}=0, \mathrm{~S}, \mathrm{Se})$ in position 4 of thieno[2,3- $d]$ pyrimidine causes the possibility of further structural modification by different reagents. Thus, as part of our ongoing research in discovery of new biologically active thienopyrimidine deriva- 
tives the aim of this work was to develop several simple and highly efficient methods of the synthesis of $\mathrm{R}_{1}, \mathrm{R}_{2}$-thieno[2,3- $\left.d\right]$ pyrimidines 3a-c and $\mathbf{4 a - c}$, the study their alkylation and antibacterial properties depending on atom in position 4 of the heterocycle.

The synthesis strategy of the starting thieno[2,3- $d$ ] pyrimidin-4(3H)-ones 1a-c was based on the classical Hewald reaction reported in the literature $[7,8]$. Treatment of ketones with ethyl 2-cyanoacetate gave the corresponding 2-aminothiophene carboxylic esters that, in turn, gave compounds 1a-c upon refluxing in formamide. Further compounds 1a-c were converted into the corresponding 4-chlorothieno[2.3- $d$ ] pyrimidines 2a-c under the action of phosphorus oxychloride and dimethylaniline. Thieno[2,3- $d]$ pyrimidine-4(3H)-thiones 3a-c and benzothieno[2,3- $d$ ] pyrimidine-4(3H)-selenones 4a-c were obtained upon treatment with such nucleophile compounds as NaHS(NaHSe) and Lawsan reagent, respectively (Scheme 1).

The presence of different reaction centres in thieno[2,3- $d$ ] pyrimidines 1a-c and 3,4a-c made possible to obtain a number of new potential biologically active compounds. The alkylation of the starting compounds $1 \mathrm{a}-\mathrm{c}$ was studied at $50-70^{\circ} \mathrm{C}$ in the presence of alkali (Scheme 2). The acidity of the NH group under these conditions provided the easy formation of the anion, which underwent alkylation.

Since substance 3a-c can exist in the form of thiole and thione, the formation of both S- and N- derivatives could be expected depending on the reaction conditions. Thus, when pyrimidine-4(3H)-thiones 3a-c are refluxed with $\mathrm{NaOH}$ and the corresponding derivatives of chloroacetic acid in ethanol, S-substituted derivatives are supposed to be obtained. However, the use of $\mathrm{NaHCO}_{3}$ and DMF as a solvent did not lead to formation of $\mathrm{N}$-substituted derivatives as it was expected. It was found that the alkylation occurred at the more nucleophilic sulphur atom in both cases (Scheme 3). All the final products were isolated as crystalline substances with a high melting point (Table 4). The structures of the compounds obtained were confirmed by IR- and NMR ${ }^{1} \mathrm{H}$ spectroscopy (Table 5).

The formation of the energetically preferable aromatic system was confirmed by the chemical shift of the signal of protons of the - $\mathrm{CH}$ group (8.7-8.9 ppm) in ${ }^{1} \mathrm{H}$ NMR spectra compared to the basic values. In the case of N-alkylation such an effect could not be observed.

All the compounds synthesized were screened for their antimicrobial and antifungal activities. The results of the antimicrobial screening by the stiff plate agar diffusion method are given in Table 1 . The results of the antimicrobial and antifungal screening by the serial dilution method are presented in Table 2 and 3. Substances that did not reveal the antimicrobial and antifungal activity were not included in Tab-

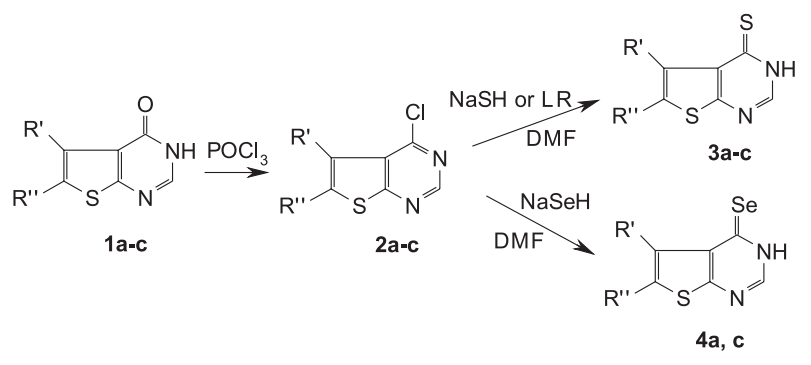

Scheme 1. The synthesis of key precursors $\mathbf{1} \mathbf{a}-\mathbf{c}-\mathbf{4} \mathbf{a}-\mathbf{b}$.

When: $a-R^{\prime}-R^{\prime \prime}=-\left(\mathrm{CH}_{2}\right)_{4}-; b-R^{\prime}-R^{\prime \prime}=-\left(\mathrm{CH}_{2}\right)_{3}{ }^{-} ; c-R^{\prime}=R^{\prime \prime}=\mathrm{CH}_{3}$.
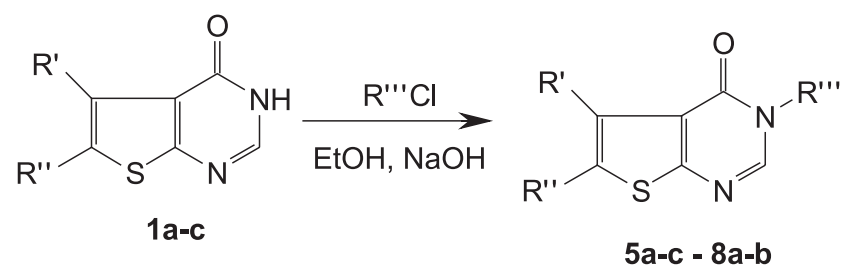

Scheme 2. Alkylation of compounds 1a-c.

When: 1a - R'=R"= -( $\left(\mathrm{CH}_{2}\right)_{4}-; 1 \mathbf{b}-\mathrm{R}^{\prime}=\mathrm{R}^{\prime \prime}=-\left(\mathrm{CH}_{2}\right)_{3}-; \mathbf{1 c}-\mathrm{R}^{\prime}=\mathrm{R}^{\prime \prime}=\mathrm{CH}_{3}$; $5 \mathbf{a}-\mathrm{R}^{\prime}=\mathrm{R}^{\prime \prime}=-\left(\mathrm{CH}_{2}\right)_{4}{ }^{-}, \mathrm{R}^{\prime \prime \prime}=-\mathrm{CH}_{2}-\mathrm{CO}-\mathrm{OC}_{2} \mathrm{H}_{5} ; \mathbf{5 b}-\mathrm{R}^{\prime}=\mathrm{R}^{\prime \prime}=-\left(\mathrm{CH}_{2}\right)_{3}{ }^{-}$,

$\mathrm{R}^{\prime \prime \prime}=-\mathrm{CH}_{2}-\mathrm{CO}-\mathrm{OC}_{2} \mathrm{H}_{5} ; \mathbf{5 c}-\mathrm{R}$ '=R" $=\mathrm{CH}_{3},-\mathrm{CH}_{2}-\mathrm{CO}-\mathrm{OC}_{2} \mathrm{H}_{5}$;

$6 \mathbf{a}-\mathrm{R}^{\prime}=\mathrm{R}^{\prime \prime}=-\left(\mathrm{CH}_{2}\right)_{4}-, \mathrm{R}^{\prime \prime}=-\mathrm{CH}_{2}-\mathrm{CO}-\mathrm{NH}-\mathrm{SO}_{2}-\mathrm{C}_{6} \mathrm{H}_{4}-\mathrm{Cl}$;

$6 \mathbf{b}-\mathrm{R}^{\prime}=\mathrm{R}^{\prime \prime}=-\left(\mathrm{CH}_{2}\right)_{3^{-}}, \mathrm{R}^{\prime \prime \prime}=-\mathrm{CH}_{2}-\mathrm{CO}-\mathrm{NH}-\mathrm{SO}_{2}-\mathrm{C}_{6} \mathrm{H}_{4}-\mathrm{Cl}$;

$6 c-\mathrm{R}^{\prime}=\mathrm{R}^{\prime \prime}=\mathrm{CH}_{3},-\mathrm{CH}_{2}-\mathrm{CO}-\mathrm{NH}-\mathrm{SO}_{2}-\mathrm{C}_{6} \mathrm{H}_{4}-\mathrm{Cl} ; 7 \mathrm{a}-\mathrm{R}^{\prime}=\mathrm{R}^{\prime \prime}=-\left(\mathrm{CH}_{2}\right)_{4}$,

$\mathrm{R}^{\prime \prime}=$ (5-isopropyl-1,3,4-thiadiazol-2-yl)-2-oxoethyl; 7b-R'=R"= -( $\left(\mathrm{CH}_{2}\right)_{3}$-,

R"'=(5-isopropyl-1,3,4-thiadiazol-2-yl)-2-oxoethyl; 7c $-\mathrm{R}^{\prime}=\mathrm{R}$ "' $=\mathrm{CH}_{3}$,

R'"=(5-isopropyl-1,3,4-thiadiazol-2-yl)-2-oxoethyl; 8a - R'=R"=

$-\left(\mathrm{CH}_{2}\right)_{4^{-}}, \mathrm{R}^{\prime \prime}=-\mathrm{CH}_{2}-\mathrm{NH}-\mathrm{CO}-\mathrm{Ph} ; \mathbf{8 b}-\mathrm{R}^{\prime}=\mathrm{R}^{\prime \prime}=-\left(\mathrm{CH}_{2}\right)_{3}{ }^{-}$,

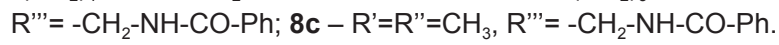

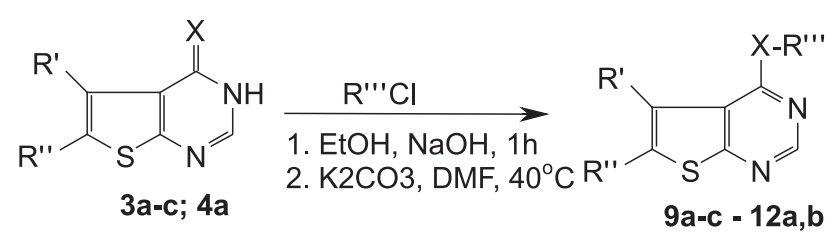

Scheme 3. Alkylation of compounds 3a-c-4a.

When: $3 a-R^{\prime}=R^{\prime \prime}=-\left(\mathrm{CH}_{2}\right)_{4^{-}}, X=S ; 3 b-R^{\prime}=R^{\prime \prime}=-\left(\mathrm{CH}_{2}\right)_{3^{-}}, X=S$;

$3 c-R^{\prime}=R^{\prime \prime}=\mathrm{CH}_{3}, \mathrm{X}=\mathrm{S} ; 4 \mathrm{a}-\mathrm{R}^{\prime}=\mathrm{R}^{\prime \prime}=-\left(\mathrm{CH}_{2}\right)_{4^{-}}, \mathrm{X}=\mathrm{Se}$;

$9 a-R^{\prime}=R^{\prime \prime}=-\left(\mathrm{CH}_{2}\right)_{4}-, \mathrm{R}^{\prime \prime}=-\mathrm{CH}_{2}-\mathrm{CO}-\mathrm{OC}_{2} \mathrm{H}_{5}, \mathrm{X}=\mathrm{S} ; \mathbf{9 b}-\mathrm{R}$ '=R" = $-\left(\mathrm{CH}_{2}\right)_{3}{ }^{-}$,

$\mathrm{R}^{\prime \prime \prime}=-\mathrm{CH}_{2}-\mathrm{CO}-\mathrm{OC}_{2} \mathrm{H}_{5}, \mathrm{X}=\mathrm{S} ; 9 \mathrm{c}-\mathrm{R}$ '=R" $=\mathrm{CH}_{3},-\mathrm{CH}_{2}-\mathrm{CO}-\mathrm{OC}_{2} \mathrm{H}_{5}, \mathrm{X}=\mathrm{S}$;

$10 \mathrm{a}-\mathrm{R}^{\prime}=\mathrm{R}^{\prime \prime}=-\left(\mathrm{CH}_{2}\right)_{4}-, \mathrm{R}^{\prime \prime}=-\mathrm{CH}_{2}-\mathrm{CO}-\mathrm{NH}-\mathrm{SO}_{2}-\mathrm{C}_{6} \mathrm{H}_{4}-\mathrm{Cl}, \mathrm{X}=\mathrm{S}$;

$10 b-R^{\prime}=R^{\prime \prime}=-\left(\mathrm{CH}_{2}\right)_{3}-, \mathrm{R}^{\prime \prime \prime}=-\mathrm{CH}_{2}-\mathrm{CO}-\mathrm{NH}-\mathrm{SO}_{2}-\mathrm{C}_{6} \mathrm{H}_{4}-\mathrm{Cl}, \mathrm{X}=\mathrm{S}$;

$10 c-R^{\prime}=R^{\prime \prime}=\mathrm{CH}_{3},-\mathrm{CH}_{2}-\mathrm{CO}-\mathrm{NH}-\mathrm{SO}_{2}-\mathrm{C}_{6} \mathrm{H}_{4}-\mathrm{Cl}, \mathrm{X}=\mathrm{S}$;

$11 \mathrm{a}-\mathrm{R}$ '=R"'=-( $\left(\mathrm{CH}_{2}\right)_{4^{-}}, \mathrm{R}^{\prime \prime}=(5$-isopropyl-1,3,4-thiadiazol-2-yl)-

2-oxoethyl, X=S; 11b - R'=R" =- $\left(\mathrm{CH}_{2}\right)_{3}-$, R'"'=(5-isopropyl-1,3,4-

thiadiazol-2-yl)-2-oxoethyl, $\mathrm{X}=\mathrm{S} ; 11 \mathrm{c}-\mathrm{R}$ '=R"= $\mathrm{CH}_{3}, \mathrm{R}$ "'=(5-isopropyl-

1,3,4-thiadiazol-2-yl)-2-oxoethyl, $\mathrm{X}=\mathrm{S} ; \mathbf{1 2 a}-\mathrm{R}^{\prime}=\mathrm{R}^{\prime \prime}=-\left(\mathrm{CH}_{2}\right)_{4^{-}}, \mathrm{X}=\mathrm{Se}$;

$\mathrm{R}^{\prime \prime}=-\mathrm{CH}_{2}-\mathrm{CO}-\mathrm{NH}-\mathrm{SO}_{2}-\mathrm{C}_{6} \mathrm{H}_{4}-\mathrm{Cl} ; 12 \mathrm{~b}-\mathrm{R}^{\prime}=\mathrm{R}$ " $=-\left(\mathrm{CH}_{2}\right)_{4^{-}}, \mathrm{X}=\mathrm{Se}$,

$\mathrm{R}^{\prime \prime}=(5$-isopropyl-1,3,4-thiadiazol-2-yl)-2-oxoethyl.

les. According to the stiff plate agar diffusion method compounds $\mathbf{4 a}$ and $\mathbf{4 c}$ revealed a significant activity against E. coli, S. aureus, and M. luteum appeared to be sensitive only to compound 10a.

The best fungistatic indicators against $\mathrm{A}$. niger were estimated for substances $\mathbf{4 a}$ and $\mathbf{9 a}$. The diameter of the inhibition zones of the microbial growth for compound $\mathbf{4 a}$ was 22.0 and $12.0 \mathrm{~mm}$ in the concentrations of 0.5 and $0.1 \%$, respectively. MIC of $\mathbf{4 a}$ and 9a were $31.2 \mu \mathrm{g} / \mathrm{mL}$. 
Table 1

The antimicrobial activity of the compounds synthesized (the stiff plate agar diffusion method)

\begin{tabular}{|c|c|c|c|c|c|}
\hline \multirow{2}{*}{ Compound $^{\text {a) }}$} & \multirow{2}{*}{ Concentration, \% } & \multicolumn{3}{|c|}{ The inhibition zones of the compounds studied (mm) } \\
\cline { 3 - 6 } & & E. coli & S. aureus & M. luteum & A. niger \\
\hline 1c & 0.5 & - & - & & 7.0 \\
\hline 2c & 0.5 & - & - & & 22.0 (f/a) \\
\hline \multirow{2}{*}{ 4a } & 0.5 & $14.0(\mathrm{~b} / \mathrm{a})$ & - & - & 12.0 (f/a) \\
\cline { 2 - 6 } & 0.1 & - & - & - & - \\
\hline \multirow{2}{*}{ 4c } & 0.5 & 14.7 & - & - & - \\
\hline 7c & 0.1 & 10.0 & - & - & - \\
\hline $\mathbf{1 0 a}$ & 0.5 & - & 12.0 & 17.0 & - \\
\hline
\end{tabular}

a)Compounds $\mathbf{1 a}, \mathbf{4 b}, \mathbf{5 a}-\mathbf{7 b}, \mathbf{8} \mathbf{a}-\mathbf{1 2} \mathbf{b}$ in the concentration of 0.5 and $1.0 \%$ did not inhibit bacteria and fungi selected; b/a - bacteriostatic action; f/a - fungistatic action; - have not been found.

Table 2

The minimal inhibitory concentration (MIC) and the minimal bactericidal concentration (MBC) of the compounds synthesized (serial dilution method)

\begin{tabular}{|c|c|c|c|c|c|c|}
\hline \multirow{2}{*}{ Compound $)$} & \multicolumn{2}{|c|}{ E. coli } & \multicolumn{2}{c|}{ S. aureus } & \multicolumn{2}{c|}{ M. luteum } \\
\cline { 2 - 7 } & $\mathrm{MIC}(\mu \mathrm{g} / \mathrm{mL})$ & $\mathrm{MBC}(\mu \mathrm{g} / \mathrm{mL})$ & $\mathrm{MIC}(\mu \mathrm{g} / \mathrm{mL})$ & $\mathrm{MBC}(\mu \mathrm{g} / \mathrm{mL})$ & $\mathrm{MIC}(\mu \mathrm{g} / \mathrm{mL})$ & $\mathrm{MBC}(\mu \mathrm{g} / \mathrm{mL})$ \\
\hline $\mathbf{1 c}$ & + & + & + & + & 250.0 & 500.0 \\
\hline $\mathbf{4 c}$ & 500.0 & $*$ & + & + & + & + \\
\hline $\mathbf{5 c}$ & + & + & + & + & 500.0 & $*$ \\
\hline $\mathbf{6 c}$ & + & + & + & + & 31.2 & 125.0 \\
\hline 7c & + & + & + & 31.2 & 125.0 \\
\hline $\mathbf{1 0 a}$ & + & + & 31.2 & 125.0 & 3 & + \\
\hline $\mathbf{1 1 a}$ & + & + & 31.2 & 62.5 & + & + \\
\hline
\end{tabular}

a)Compounds $\mathbf{1 a}-\mathbf{b}, \mathbf{2} \mathbf{a}-\mathbf{4} \mathbf{b}, \mathbf{5} \mathbf{a}-\mathbf{b}, \mathbf{6} \mathbf{\mathbf { a }}-\mathbf{b}, \mathbf{7} \mathbf{a}-\mathbf{b}, \mathbf{8} \mathbf{a}-\mathbf{9} \mathbf{c}, \mathbf{1 0 b}-\mathbf{c}, \mathbf{1 1} \mathbf{b}-\mathbf{1 2} \mathbf{b}$ did not reveal the antibacterial activity in the concentrations tested; b) + The antibacterial activity was not observed in the concentrations tested; c) $*$ The antibacterial activity was not determined in the concentrations tested.

Table 3

The minimal inhibitory concentration (MIC) and the minimal fungicidal concentration (MFC) of the compounds synthesized (serial dilution method)

\begin{tabular}{|c|c|c|c|c|}
\hline \multirow{2}{*}{ Compound ${ }^{a)}$} & \multicolumn{2}{|c|}{ Candida tenus } & \multicolumn{2}{|c|}{ Aspergillus niger } \\
\hline & $\mathrm{MIC}(\mu \mathrm{g} / \mathrm{mL})$ & $\mathrm{MFC}(\mu \mathrm{g} / \mathrm{mL})$ & $\mathrm{MIC}(\mu \mathrm{g} / \mathrm{mL})$ & $\mathrm{MFC}(\mu \mathrm{g} / \mathrm{mL})$ \\
\hline 1c & 125.0 & 250.0 & 125.0 & * \\
\hline $3 c$ & + & + & 62.5 & 250.0 \\
\hline $4 a$ & + & + & 31.2 & * \\
\hline $5 b$ & + & + & 250.0 & 500.0 \\
\hline $5 c$ & + & + & 500.0 & * \\
\hline $6 c$ & 125.0 & * & 500.0 & * \\
\hline $8 a$ & 250.0 & * & 500.0 & * \\
\hline $9 a$ & + & + & 31.2 & * \\
\hline
\end{tabular}

a) Compounds 1a-b, 2a-3b, 4b-c, 5a, 6a-b, 7a-c, 8b-c, 9b-c, 10a-12b did not reveal the antifungal activity in the concentrations tested;

b) * The antifungal activity was not determined in the concentrations tested; $c)+$ The antifungal activity was not observed in the concentrations tested. 
Table 4

Physicochemical properties of alkylation compounds $\mathbf{5 a - 1 2 b}$

\begin{tabular}{|c|c|c|c|c|c|c|c|c|c|}
\hline \multirow{2}{*}{$\begin{array}{l}\text { Compd } \\
\text { No }\end{array}$} & \multirow{2}{*}{ Yield, \% } & \multirow{2}{*}{ M.p., ${ }^{\circ} \mathrm{C}$} & \multicolumn{3}{|c|}{ Found, \% } & \multirow{2}{*}{$\begin{array}{l}\text { Mol. formula } \\
\text { M.w }\end{array}$} & \multicolumn{3}{|c|}{ Calc, \% } \\
\hline & & & C & $\mathrm{H}$ & $\mathrm{N}$ & & C & $\mathrm{H}$ & $\mathrm{N}$ \\
\hline $5 a$ & 77 & 276 & 57.52 & 5.56 & 9.58 & $\mathrm{C}_{14} \mathrm{H}_{16} \mathrm{~N}_{2} \mathrm{O}_{3} \mathrm{~S}$ & 57.52 & 5.52 & 9.58 \\
\hline $5 b$ & 87 & 236 & 56.12 & 5.10 & 10.09 & $\mathrm{C}_{13} \mathrm{H}_{14} \mathrm{~N}_{2} \mathrm{O}_{3} \mathrm{~S}$ & 56.10 & 5.07 & 10.06 \\
\hline $5 c$ & 83 & 281 & 54.12 & 5.27 & 10.50 & $\mathrm{C}_{12} \mathrm{H}_{14} \mathrm{~N}_{2} \mathrm{O}_{3} \mathrm{~S}$ & 54.12 & 5.30 & 10.52 \\
\hline $6 a$ & 81 & 297 & 49.36 & 3.66 & 9.58 & $\mathrm{C}_{18} \mathrm{H}_{16} \mathrm{ClN}_{3} \mathrm{O}_{4} \mathrm{~S}$ & 49.37 & 3.68 & 9.60 \\
\hline $6 \mathbf{b}$ & 59 & 251 & 49.71 & 3.69 & 6.82 & $\mathrm{C}_{17} \mathrm{H}_{14} \mathrm{ClN}_{3} \mathrm{O}_{4} \mathrm{~S}$ & 49.69 & 3.68 & 6.82 \\
\hline $6 c$ & 79 & 312 & 48.21 & 3.36 & 9.89 & $\mathrm{C}_{14} \mathrm{H}_{16} \mathrm{ClN}_{3} \mathrm{O}_{4} \mathrm{~S}$ & 48.17 & 3.33 & 9.91 \\
\hline $7 a$ & 71 & 307 & 55.68 & 5.19 & 14.43 & $\mathrm{C}_{18} \mathrm{H}_{20} \mathrm{~N}_{4} \mathrm{O}_{2} \mathrm{~S}_{2}$ & 55.65 & 5.19 & 14.42 \\
\hline $7 \mathrm{~b}$ & 67 & 257 & 54.52 & 4.85 & 14.96 & $\mathrm{C}_{17} \mathrm{H}_{18} \mathrm{~N}_{4} \mathrm{O}_{2} \mathrm{~S}_{2}$ & 54.53 & 4.84 & 14.96 \\
\hline $7 c$ & 86 & 327 & 53.05 & 5.02 & 15.47 & $\mathrm{C}_{16} \mathrm{H}_{18} \mathrm{~N}_{4} \mathrm{O}_{2} \mathrm{~S}_{2}$ & 53.02 & 5.01 & 15.46 \\
\hline $8 a$ & 68 & 286 & 63.74 & 5.07 & 12.41 & $\mathrm{C}_{18} \mathrm{H}_{17} \mathrm{~N}_{3} \mathrm{O}_{2} \mathrm{~S}$ & 63.70 & 5.05 & 12.38 \\
\hline $8 b$ & 92 & 298 & 61.36 & 4.85 & 13.41 & $\mathrm{C}_{16} \mathrm{H}_{15} \mathrm{~N}_{3} \mathrm{O}_{2} \mathrm{~S}$ & 61.32 & 4.82 & 13.41 \\
\hline $9 a$ & 57 & 231 & 54.57 & 5.23 & 9.11 & $\mathrm{C}_{14} \mathrm{H}_{16} \mathrm{~N}_{2} \mathrm{O}_{2} \mathrm{~S}_{2}$ & 54.52 & 5.23 & 9.08 \\
\hline $9 b$ & 72 & 245 & 63.74 & 5.07 & 12.41 & $\mathrm{C}_{13} \mathrm{H}_{14} \mathrm{~N}_{2} \mathrm{O}_{2} \mathrm{~S}_{2}$ & 63.70 & 5.05 & 12.38 \\
\hline $9 c$ & 65 & 261 & 51.09 & 5.07 & 9.93 & $\mathrm{C}_{12} \mathrm{H}_{14} \mathrm{~N}_{2} \mathrm{O}_{2} \mathrm{~S}_{2}$ & 51.04 & 5.00 & 9.92 \\
\hline $10 a$ & 77 & 279 & 46.43 & 3.14 & 9.59 & $\mathrm{C}_{17} \mathrm{H}_{14} \mathrm{CIN}_{3} \mathrm{O}_{3} \mathrm{~S}_{3}$ & 46.41 & 3.21 & 9.55 \\
\hline $11 a$ & 69 & 277 & 53.20 & 5.48 & 13.80 & $\mathrm{C}_{18} \mathrm{H}_{22} \mathrm{~N}_{4} \mathrm{OS}_{3}$ & 53.17 & 5.45 & 13.78 \\
\hline $11 \mathrm{~b}$ & 83 & 289 & 52.04 & 5.14 & 14.30 & $\mathrm{C}_{17} \mathrm{H}_{20} \mathrm{~N}_{4} \mathrm{OS}_{3}$ & 52.01 & 5.14 & 14.27 \\
\hline $11 \mathrm{c}$ & 75 & 269 & 50.55 & 5.34 & 14.75 & $\mathrm{C}_{16} \mathrm{H}_{20} \mathrm{~N}_{4} \mathrm{OS}_{3}$ & 50.50 & 5.30 & 14.72 \\
\hline $12 a$ & 68 & 286 & 60.82 & 4.82 & 11.82 & $\mathrm{C}_{18} \mathrm{H}_{16} \mathrm{CIN}_{3} \mathrm{O}_{3} \mathrm{~S}_{2} \mathrm{Se}$ & 60.82 & 4.82 & 11.82 \\
\hline $12 b$ & 52 & 256 & 47.94 & 4.45 & 12.38 & $\mathrm{C}_{18} \mathrm{H}_{20} \mathrm{CIN}_{4} \mathrm{OS}_{2} \mathrm{Se}$ & 47.89 & 4.47 & 12.41 \\
\hline
\end{tabular}

Table 5

The data of IR and ${ }^{1} \mathrm{H}$ NMR spectral of alkylation compounds $5 a-12 b$

\begin{tabular}{|c|c|c|c|c|c|}
\hline Compd & IR ATR, $\mathrm{cm}^{-1}$ & $\mathrm{NH}$ & Pyrimidine $\mathrm{CH}$ & Aliphatic protons & Aromatic protons \\
\hline 1 & 2 & 3 & 4 & 5 & 6 \\
\hline $5 \mathbf{a}$ & & & $8.3(1 \mathrm{H}, \mathrm{s}, \mathrm{CH})$ & $\begin{array}{l}4.77\left(2 \mathrm{H}, s, \mathrm{CH}_{2}\right) ; 4.17-4.22(2 \mathrm{H}, q \\
\left.J=6.95 \mathrm{~Hz}_{2} \mathrm{CH}_{2}\right) ; 2.9-2.5\left(8 \mathrm{H}, m_{1}\right. \\
\left.4 \mathrm{CH}_{2}\right) ; 1.3\left(3 \mathrm{H}, t, J=6.95 \mathrm{~Hz}_{3} \mathrm{CH}_{3}\right)\end{array}$ & \\
\hline $5 \mathbf{b}$ & & & $8.3(1 \mathrm{H}, \mathrm{s}, \mathrm{CH})$ & $\begin{array}{l}4.7\left(2 \mathrm{H}, \mathrm{s}, \mathrm{CH}_{2}\right) ; 4.11-4.12(2 \mathrm{H}, q \\
\left.J=6.99 \mathrm{~Hz}, \mathrm{CH}_{2}\right) ; 2.9-2.8(4 \mathrm{H}, m \\
\left.2 \mathrm{CH}_{2}\right) ; 2.4-2.3\left(2 \mathrm{H}, \mathrm{m}_{1} \mathrm{CH}_{2}\right) ; 1.17- \\
1.24\left(3 \mathrm{H}, \mathrm{t}, J=6.94 \mathrm{~Hz}, \mathrm{CH}_{3}\right)\end{array}$ & \\
\hline $5 c$ & & & $8.3(1 \mathrm{H}, \mathrm{s}, \mathrm{CH})$ & $\begin{array}{l}\text { 4.7 }\left(2 \mathrm{H}, s, \mathrm{CH}_{2}\right) ; 4.14-4.18(2 \mathrm{H}, q \\
\left.J=7.0 \mathrm{~Hz}_{1} \mathrm{CH}_{2}\right) ; 2.37\left(6 \mathrm{H}, \mathrm{s}, 2 \mathrm{CH}_{3}\right) ; \\
\text { 1.18-1.24 }\left(3 \mathrm{H}, \mathrm{t}, \mathrm{J}=6.97 \mathrm{~Hz} \mathrm{CH}_{3}\right)\end{array}$ & \\
\hline $6 a$ & & $12.6(1 \mathrm{H}, \mathrm{s}, \mathrm{NH})$ & $8.17(1 \mathrm{H}, s, \mathrm{CH})$ & $\begin{array}{l}4.69\left(2 \mathrm{H}, s, \mathrm{CH}_{2}\right) ; 2.74-2-2.68(4 \mathrm{H}, \\
\left.m, 2 \mathrm{CH}_{2}\right) ; 1.74-1.66\left(4 \mathrm{H}, m, 2 \mathrm{CH}_{2}\right)\end{array}$ & $\begin{array}{l}7.91(2 \mathrm{H}, d, J=8.78 \mathrm{~Hz}, \\
\mathrm{Ar}-\mathrm{H}) ; 7.72(2 \mathrm{H}, d, \\
J=8.42 \mathrm{~Hz}, \mathrm{Ar}-\mathrm{H})\end{array}$ \\
\hline 6b & & $12.6(1 \mathrm{H}, \mathrm{s}, \mathrm{NH})$ & $8.3(1 \mathrm{H}, \mathrm{s}, \mathrm{CH})$ & $\begin{array}{l}4.6\left(2 \mathrm{H}, s, \mathrm{CH}_{2}\right) ; 2.75-2.9(4 \mathrm{H}, m, \\
\left.2 \mathrm{CH}_{2}\right) ; 2.3-2.4\left(2 \mathrm{H}, m, \mathrm{CH}_{2}\right)\end{array}$ & $\begin{array}{l}7.92(2 \mathrm{H}, d, J=8.74 \\
\mathrm{Hz}, \mathrm{Ar}-\mathrm{H}) ; 7.7(2 \mathrm{H}, d \\
J=8.79 \mathrm{~Hz}, \mathrm{Ar}-\mathrm{H})\end{array}$ \\
\hline $6 c$ & & $12.6(1 \mathrm{H}, \mathrm{s}, \mathrm{NH})$ & $8.17(1 \mathrm{H}, \mathrm{s}, \mathrm{CH})$ & $\begin{array}{l}4.7\left(2 \mathrm{H}, s, \mathrm{CH}_{2}\right) ; 2.31-2.33(6 \mathrm{H}, \mathrm{s}, \\
\left.2 \mathrm{CH}_{3}\right)\end{array}$ & $\begin{array}{l}7.91(2 \mathrm{H}, d, J=8.74 \\
\mathrm{Hz}, \mathrm{Ar}-\mathrm{H}) ; 7.70(2 \mathrm{H}, d, \\
J=8.74 \mathrm{~Hz}, \mathrm{Ar}-\mathrm{H})\end{array}$ \\
\hline $7 a$ & & $12.95(1 \mathrm{H}, \mathrm{s}, \mathrm{NH})$ & $8.3(1 \mathrm{H}, s, \mathrm{CH})$ & $\begin{array}{l}4.94\left(2 \mathrm{H}, s, \mathrm{CH}_{2}\right) ; 2.75-2.82(5 \mathrm{H} \\
\left.m, 2 \mathrm{CH}_{2}\right) ; 1.76-1.64\left(4 \mathrm{H}, m, 2 \mathrm{CH}_{2}\right) ; \\
1.3\left(6 \mathrm{H}, d, J=6.4 \mathrm{~Hz}, 2 \mathrm{CH}_{3}\right)\end{array}$ & \\
\hline
\end{tabular}


Continuation Table 5

\begin{tabular}{|c|c|c|c|c|c|}
\hline 1 & 2 & 3 & 4 & 5 & 6 \\
\hline $7 \mathbf{b}$ & & $12.96(1 \mathrm{H}, \mathrm{s}, \mathrm{NH})$ & $8.3(1 \mathrm{H}, \mathrm{s}, \mathrm{CH})$ & $\begin{array}{l}4.96\left(2 \mathrm{H}, s, \mathrm{CH}_{2}\right) ; 2.72-2.88(5 \mathrm{H}, \\
\left.m, 2 \mathrm{CH}_{2}\right) ; 2.35-2.4\left(2 \mathrm{H}, m_{,} \mathrm{CH}_{2}\right) ; \\
1.31\left(6 \mathrm{H}, d, J=6.49 \mathrm{~Hz}, 2 \mathrm{CH}_{3}\right)\end{array}$ & \\
\hline 7c & & $12.94(1 \mathrm{H}, \mathrm{s}, \mathrm{NH})$ & $8.3(1 \mathrm{H}, \mathrm{s}, \mathrm{CH})$ & $\begin{array}{l}4.94\left(2 \mathrm{H}, s, \mathrm{CH}_{2}\right) ; 2.35(6 \mathrm{H}, s, \\
\left.2 \mathrm{CH}_{3}\right) ; 1.32(6 \mathrm{H}, d, J=6.51 \mathrm{~Hz}, \\
\left.2 \mathrm{CH}_{3}\right)\end{array}$ & \\
\hline $8 a$ & & $9.6(1 \mathrm{H}, b r s, \mathrm{NH})$ & $8.48(1 \mathrm{H}, \mathrm{s}, \mathrm{CH})$ & $\begin{array}{l}5.4\left(2 \mathrm{H}, d, J=6.58 \mathrm{~Hz}, \mathrm{CH}_{2}\right) ; 2.72- \\
2.88\left(4 \mathrm{H}, \mathrm{m}, 2 \mathrm{CH}_{2}\right) ; 1.7-1.83(4 \mathrm{H}, \\
\left.\mathrm{m}, 2 \mathrm{CH}_{2}\right)\end{array}$ & $\begin{array}{l}7.88(2 \mathrm{H}, m, \mathrm{Ar}-\mathrm{H}) \\
7.5(3 \mathrm{H}, m, \mathrm{Ar}-\mathrm{H})\end{array}$ \\
\hline $8 b$ & & $9.39(1 \mathrm{H}, b r s, \mathrm{NH})$ & $7.98(1 \mathrm{H}, \mathrm{s}, \mathrm{CH})$ & $\begin{array}{l}4.7\left(2 \mathrm{H}, d, J=6.56 \mathrm{~Hz}, \mathrm{CH}_{2}\right) ; 2.34 \\
\left(6 \mathrm{H}, \mathrm{s}, 2 \mathrm{CH}_{3}\right)\end{array}$ & $\begin{array}{l}7.8(2 \mathrm{H}, m, \mathrm{Ar}-\mathrm{H}) \\
7.5(3 \mathrm{H}, m, \mathrm{Ar}-\mathrm{H})\end{array}$ \\
\hline $9 a$ & $\begin{array}{c}2985,2937,2857 \\
2751,2674,1413 \\
1257,1071,809 \\
732\end{array}$ & & $8.65(1 \mathrm{H}, \mathrm{s}, \mathrm{CH})$ & $\begin{array}{l}4.16-4.14\left(4 \mathrm{H}, m, 2 \mathrm{CH}_{2}\right) ; 2.8-3.0 \\
\left(4 \mathrm{H}, m, 2 \mathrm{CH}_{2}\right) ; 1.6-1.83(4 \mathrm{H}, m, \\
\left.2 \mathrm{CH}_{2}\right) ; 1.14-1.21(3 \mathrm{H}, t, J=6.99 \mathrm{~Hz}, \\
\left.\mathrm{CH}_{3}\right)\end{array}$ & \\
\hline $9 b$ & $\begin{array}{c}2999,2974,2908 \\
2853,1744,1667 \\
1452,1175,1086 \\
853,781,742\end{array}$ & & $8.3(1 \mathrm{H}, \mathrm{s}, \mathrm{CH})$ & $\begin{array}{l}4.79\left(2 \mathrm{H}, \mathrm{s}, \mathrm{CH}_{2}\right) ; 4.14-4.19(2 \mathrm{H}, q, \\
\left.J=7.12 \mathrm{~Hz}, \mathrm{CH}_{2}\right) ; 2.9-3.07(4 \mathrm{H}, m, \\
\left.2 \mathrm{CH}_{2}\right) ; 2.37-2.42\left(2 \mathrm{H}, m, \mathrm{CH}_{2}\right) ; 1.1 \\
\left(3 \mathrm{H}, t, J=6.95 \mathrm{~Hz}, \mathrm{CH}_{3}\right)\end{array}$ & \\
\hline $9 c$ & $\begin{array}{c}2978,2908,2860 \\
1738,1535,1166, \\
882,629 \\
\end{array}$ & & $8.63(1 \mathrm{H}, \mathrm{s}, \mathrm{CH})$ & $\begin{array}{l}4.03-4.13\left(4 \mathrm{H}, m, 2 \mathrm{CH}_{2}\right) ; 2.44(6 \mathrm{H}, \\
\left.s, 2 \mathrm{CH}_{3}\right) ; 1.11-1.18(3 \mathrm{H}, t, J=6.99 \\
\left.\mathrm{Hz} \mathrm{CH}_{3}\right)\end{array}$ & \\
\hline $10 a$ & & $12.3(1 \mathrm{H}, \mathrm{s}, \mathrm{NH})$ & $8.5(1 \mathrm{H}, \mathrm{s}, \mathrm{CH})$ & $\begin{array}{l}4.1\left(2 \mathrm{H}, s, \mathrm{CH}_{2}\right) ; 3.0-3.2(4 \mathrm{H}, m, \\
\left.2 \mathrm{CH}_{2}\right) ; 2.1-2.3\left(2 \mathrm{H}, m, \mathrm{CH}_{2}\right)\end{array}$ & $\begin{array}{l}7.8\left(3 \mathrm{H}, m, \mathrm{CH}_{\mathrm{ar}}\right) \\
7.4\left(2 \mathrm{H}, m, \mathrm{CH}_{\mathrm{ar}}\right)\end{array}$ \\
\hline $11 \mathrm{a}$ & & $12.6(1 \mathrm{H}, \mathrm{s}, \mathrm{NH})$ & $7.94(1 \mathrm{H}, \mathrm{s}, \mathrm{CH})$ & $\begin{array}{l}4.37\left(2 \mathrm{H}, s, \mathrm{CH}_{2}\right) ; 2.77-2.88(4 \mathrm{H}, \\
\left.m, 2 \mathrm{CH}_{2}\right) ; 2.0-2.06\left(5 \mathrm{H}, m, 2 \mathrm{CH}_{2}\right) ; \\
1.3\left(6 \mathrm{H}, d, J=6.99 \mathrm{~Hz}, 2 \mathrm{CH}_{3}\right)\end{array}$ & \\
\hline $11 b$ & $\begin{array}{c}2964,2931,2901 \\
2858,1461,1235 \\
829,749 \\
\end{array}$ & $12.9(1 \mathrm{H}, \mathrm{s}, \mathrm{NH})$ & $8.66(1 \mathrm{H}, \mathrm{s}, \mathrm{CH})$ & $\begin{array}{l}4.4\left(2 \mathrm{H}, s, \mathrm{CH}_{2}\right) ; 3.04-3.10(7 \mathrm{H}, \\
\left.m, 3 \mathrm{CH}_{2}\right) ; 1.3(6 \mathrm{H}, d, J=6.59 \mathrm{~Hz}, \\
\left.2 \mathrm{CH}_{3}\right)\end{array}$ & \\
\hline $11 \mathrm{c}$ & $\begin{array}{c}3047,2984,2821 \\
2705,1518,1236, \\
924,838 \\
\end{array}$ & $12.8(1 \mathrm{H}$, brs, $\mathrm{NH})$ & $8.38(1 \mathrm{H}, \mathrm{s}, \mathrm{CH})$ & $\begin{array}{l}4.38\left(2 \mathrm{H}, \mathrm{s}, \mathrm{CH}_{2}\right) ; 2.55(6 \mathrm{H}, \mathrm{s}, \\
\left.2 \mathrm{CH}_{3}\right) ; 1.3(6 \mathrm{H}, d, J=6.99 \mathrm{~Hz}, \\
\left.2 \mathrm{CH}_{3}\right)\end{array}$ & \\
\hline $12 a$ & & $12.5(1 \mathrm{H}, \mathrm{s}, \mathrm{NH})$ & $8.4(1 \mathrm{H}, \mathrm{s}, \mathrm{CH})$ & $\begin{array}{l}4.07\left(2 \mathrm{H}, s, \mathrm{CH}_{2}\right) ; 2.82-2.92(4 \mathrm{H}, \\
\left.m, 2 \mathrm{CH}_{2}\right) ; 1.74-1.83\left(4 \mathrm{H}, m, 2 \mathrm{CH}_{2}\right)\end{array}$ & $\begin{array}{l}7.9(2 \mathrm{H}, d, J=8.78 \mathrm{~Hz}, \\
\operatorname{Ar}-\mathrm{H}) ; 7.5(2 \mathrm{H}, d, \\
J=8.42 \mathrm{~Hz}, \mathrm{Ar}-\mathrm{H})\end{array}$ \\
\hline $12 b$ & $\begin{array}{c}2929,2867,2834 \\
2696,1408,1316, \\
1152,1076,769 \\
618\end{array}$ & $12.75(1 \mathrm{H}, \mathrm{s}, \mathrm{NH})$ & $8.66(1 \mathrm{H}, \mathrm{s}, \mathrm{CH})$ & $\begin{array}{l}4.36\left(2 \mathrm{H}, s, \mathrm{CH}_{2}\right) ; 2.82-2.98(5 \mathrm{H}, \\
\left.m, 2 \mathrm{CH}_{2}\right) ; 1.8-1.7\left(4 \mathrm{H}, m, 2 \mathrm{CH}_{2}\right) ; \\
1.1\left(6 \mathrm{H}, d, J=6.62 \mathrm{~Hz}, 2 \mathrm{CH}_{3}\right)\end{array}$ & \\
\hline
\end{tabular}

According to the serial dilution method the growth of $S$. aureus was mostly inhibited by compounds 10a and 11a in the concentrations of $31.2 \mu \mathrm{g} / \mathrm{mL}$. E. coli appeared to be almost insensitive to the compounds synthesized in the concentrations tested. Only compounds $4 \mathrm{c}$ revealed a weak inhibitory activity against it $(\mathrm{MIC}=500.0 \mu \mathrm{g} / \mathrm{mL}$ ). Compounds 7c and 10a had the moderate antibacterial activity against $M$. luteum (MIC $=31.2 \mu \mathrm{g} / \mathrm{mL}$ ).

\section{Experimental Part}

\section{Chemical Part}

The ${ }^{1} \mathrm{H}$ NMR spectra were recorded in DMSO- $\mathrm{d}_{6}$ on a Varian Mercury VX-200 instrument (200 MHz). The internal standard for the NMR spectra was TMS. IR spectra (4000-600 $\left.\mathrm{cm}^{-1}\right)$ were recorded on a Bruker ALPHA
FTIR spectrometer using a module for measuring attenuated total reflection (ATR) on a Bruker Tensor 27 spectrometer $(\mathrm{KBr})$ and expressed in $\mathrm{cm}^{-1}$. The mass spectra were recorded on a Varian $1200 \mathrm{~L}$ instrument with direct injection of the sample at $250^{\circ} \mathrm{C}$ and $70 \mathrm{eV}$ ionizing electrons. Elemental analysis was performed on an LECO CHNS-900 instrument. Melting points were determined in open capillary tubes and were uncorrected. The reaction and the purity of the compounds obtained were monitored by TLC on Merck Silicagel $60 \mathrm{~F}-254$ plates with $10: 1 \mathrm{CHCl}_{3}-2-\mathrm{PrOH}$ as an eluent.

Compounds 1a-c and 2a-c were synthesized by the method described in the literature $[7,8]$.

5,6-Dimethylthieno[2,3-d]pyrimidin-4(3H)one 1c: A white powder. M.p. $-268^{\circ} \mathrm{C}$. yield $-90 \%$; 
${ }^{1} \mathrm{H}$ NMR: 12.3 (1H, br s, N-H). $8.0(1 \mathrm{H}$, br s, =C-H), 2.31 (3H, s, CH3), 2.34 (3H, s, CH3); Found, \%: C 53.33; H 4.47; N 15.54. C8H8N2OS. Calculated, \%: C 53.31; H 4.47; N 15.54 .

The general procedure of the synthesis of compounds 2a-c: Dissolve compounds 1a-c (12 g) in phosphorus oxychloride, and then add gradually dimethylaniline $14 \mathrm{ml}(0.11 \mathrm{Mol})$. Heat the reaction mixture for $4 \mathrm{~h}$, evaporate the excess of phosphorous oxychloride under reduced pressure, neutralize the solid residue was with $5 \%$ aq $\mathrm{Na}_{2} \mathrm{CO}_{3}$ solution. Filter the precipitate formed and recrystallize from toluene.

4-Chloro-5,6-dimethylthieno[2,3-d]pyrimidine 2c: a light yellow powder, yield $-96 \%$, calculated for the structure proposed. M.p. $-117^{\circ} \mathrm{C}$. This intermediate chlorpyrimidine was used at the next step without purification.

The general procedure of the synthesis of compound 3a-c - method A: Dissolve compounds 2a-c ( $0.0084 \mathrm{Mol})$ in DMF, add the solution of sodium hydrosulfide (1 g, $0.0179 \mathrm{Mol}$ ). Stir the mixture obtained for $24 \mathrm{~h}$ at room temperature, adjust $\mathrm{pH}$ to $\sim 7.0$ with aq $\mathrm{NaOH}$ solution $(15 \mathrm{~mL})$. Add hydrochloric acid to the filtrate and recrystallize the white precipitate obtained from $\mathrm{CH}_{3} \mathrm{CN}$.

Method B: Reflux the mixture of compounds 2a-c $(0.0084 \mathrm{Mol})$ and the Lawsan reagent $(0.0084 \mathrm{Mol})$ in $15 \mathrm{~mL}$ of 1,4-dioxan for $6 \mathrm{~h}$ and filter hot. Recrystallize the precipitate obtained from $\mathrm{CH}_{3} \mathrm{CN}$.

5,6,7,8-Tetrahydro[1]benzothieno[2,3-d]pyrimidine-4(3H)-thione (3a): A yellow powder. Yield - 75\%. M.p. $-213^{\circ} \mathrm{C} ;{ }^{1} \mathrm{H}$ NMR: 13.5 (1H, br s, NH). 8.0 $(1 \mathrm{H}, b r s, \mathrm{CH}), 2.8-2.9\left(4 \mathrm{H}, m, 2 \mathrm{CH}_{2}\right), 1.6-1.9(4 \mathrm{H}, m$, $\left.2 \mathrm{CH}_{2}\right)$. MS: m/z (I,\%) - 222 (96\%) [M] $]^{+}$. Found, \%: C 54.05; H 4.52; N 12.60. $\mathrm{C}_{10} \mathrm{H}_{10} \mathrm{~N}_{2} \mathrm{~S}_{2}$. Calculated, \%: C 54.02; H 4.53; N 12.60 .

$3,5,6,7-T e t r a h y d r o-4 H-c y c l o p e n t a[4,5]$ thieno[2,3-d]pyrimidine-4-thione (3b): A yellow powder. Yield - 60\%. M.p. - 223-224 ${ }^{\circ} \mathrm{C} ;{ }^{1} \mathrm{H}$ NMR: $12.6(1 \mathrm{H}$, br $s, \mathrm{~N}-\mathrm{H})$. $7.9(1 \mathrm{H}, b r s, \mathrm{C}-\mathrm{H}), 2.9-3.0\left(2 \mathrm{H}, m, \mathrm{CH}_{2}\right)$, 2.0-2.2 (4H, $\left.m, 2 \mathrm{CH}_{2}\right)$; MS: $\mathrm{m} / \mathrm{z}(\mathrm{I}, \%)-208(77 \%)$ $[\mathrm{M}]^{+}$. Found, \%: C 51.91; H 3.87; N 13.45. $\mathrm{C}_{9} \mathrm{H}_{8} \mathrm{~N}_{2} \mathrm{~S}_{2}$. Calculated, \%: C 51.90; H 3.87; N 13.45;

5,6-Dimethylthieno[2,3-d]pyrimidine-4(3H)thione (3c): A yellow powder. Yield - 62\%. M.p. - 239$240^{\circ} \mathrm{C}$; ${ }^{1} \mathrm{H}$ NMR: 13.0 (1H, br s, NH). 8.4 (1H, br s, CH), 2.1 $\left(3 \mathrm{H}, s, \mathrm{CH}_{3}\right), 2.4\left(3 \mathrm{H}, s, \mathrm{CH}_{3}\right) . \mathrm{MS}: \mathrm{m} / \mathrm{z}(\mathrm{I}, \%)-208(77 \%)$ $[\mathrm{M}]^{+}$. Found, \%: C 48.95; H 4.11; N 14.25. C8H8N2S2. Calculated, \%: C 48.95; H 4.11; N 14.27.

The general procedure of the synthesis of compound 4a-c: Add selenium (0.0012 Mol) gradually to absolute ethanol (50 mL) with stirring on an ice bath. After that gradually add sodium hydroborate $0.4 \mathrm{~g}$ $(0.0032 \mathrm{Mol})$ to the solution for $15 \mathrm{~min}$. Stir the resulting mixture for $1.5 \mathrm{~h}$ until selenium is completely dissolved. Dissolve compounds 2a-c in ethanol, cool and add to the solution of sodium hydroselenide.
Stir the reaction mixture under nitrogen for $3 \mathrm{~h}$. Then pour the solution into the mixture of hydrochloric acid $(100 \mathrm{~mL})$ and ice $(50 \mathrm{~mL})$. Filter the precipitate formed and recrystallize from aq $\mathrm{MeOH}$.

5,6,7,8-Tetrahydro[1]benzothieno[2,3-d]pyrimidine-4(3H)-selenone (4a): A yellow powder. Yield - 65\%. M.p. $-190^{\circ} \mathrm{C} ;{ }^{1} \mathrm{H}$ NMR: 12.9 (1H, s, NH); $8.1(1 \mathrm{H}, s, \mathrm{CH}) ; 4.6\left(2 \mathrm{H}, s, \mathrm{CH}_{2}\right) ; 2.8-3.0\left(4 \mathrm{H}, m, 2 \mathrm{CH}_{2}\right)$. Found, \%: C 44.62; H 3.76; N 10.42. $\mathrm{C}_{10} \mathrm{H}_{10} \mathrm{~N}_{2}$ SSe. Calculated, \%: C 44.61; H 3.74; N 10.41.

5,6-Dimethylthieno[2,3-d]pyrimidine-4(3H)-selenone (4c): A yellow powder. Yield - 55\%. M.p. $-200^{\circ} \mathrm{C}$; IR (ATR, $\mathrm{cm}^{-1}$ ): 3116, 3036, 2965, 2910, 2840, 2766, $1679,1563,1485,1406,1352,1294,1212,1144,1040$, 925, 812, 705, 604. 1H NMR: 13.1 (1H, s, NH); 8.0 $(1 \mathrm{H}, s, \mathrm{CH}) ; 2.9\left(3 \mathrm{H}, s, \mathrm{CH}_{3}\right) ; 2.2(3 \mathrm{H}, s, \mathrm{CH} 3) . \mathrm{MS}: \mathrm{m} / \mathrm{z}$ $(\mathrm{I}, \%)-243$ (85\%) [M] $]^{+}$. Found, \%: C 39.51; H 3.34; $\mathrm{N}$ 11.54. $\mathrm{C}_{8} \mathrm{H}_{8} \mathrm{~N}_{2} \mathrm{SSe}$. Calculated, \%: C 39.51; H 3.32; N 11.52 .

The general procedure of alkylation of compounds 1a-c: To the suspension of compound 1a-c $(0.0044 \mathrm{Mol})$ in ethanol $(5 \mathrm{ml})$ add $0.1 \mathrm{~g}(0.0044 \mathrm{Mol})$ of sodium hydroxide and heat for $10 \mathrm{~min}$. When compounds are dissolved, add the corresponding alkylating agent $(0.0044 \mathrm{Mol})$, and reflux the mixture for 30-40 min at $70^{\circ} \mathrm{C}$. Filter the precipitate formed and recrystallize from aq $\mathrm{MeOH}$.

The general procedure of alkylation of compounds 3a-c - method A: Heat the mixture of compounds 3a-c $(0.0044 \mathrm{Mol})$ and potassium carbonate $0.8 \mathrm{~g}$ $(0.0061 \mathrm{Mol})$ in DMF $(5 \mathrm{ml})$ at $40^{\circ} \mathrm{C}$ for $5 \mathrm{~min}$. Then add the corresponding alkylhalogenide (0.0044 Mol). Stir the reaction mixture at $40^{\circ} \mathrm{C}$ for $24 \mathrm{~h}$ and pour into water. Filter the precipitate formed, dry and recrystallize from EtOH.

Method B: Add while stirring the solution of compounds 3a-c $(0.0044 \mathrm{Mol})$ in ethanol $(5 \mathrm{ml})$ to $0.2 \mathrm{~g}$ $(0.0057 \mathrm{Mol})$ of the solution of sodium hydroxide in ethanol (3 ml). Then add the corresponding alkylhalogenide $(0.0044 \mathrm{Mol})$ and reflux at $70^{\circ} \mathrm{C}$ for $30 \mathrm{~min}$. Cool the reaction mixture, filter the precipitate formed, dry and recrystallize from $\mathrm{CH}_{3} \mathrm{CN}$.

The general procedure of alkylation of compounds 4a-b: Add while stirring the solution of compounds $4 a-c(0.0044 \mathrm{Mol})$ in ethanol $(5 \mathrm{ml})$ to the solution of sodium hydroxide (0.0057 Mol) in ethanol ( $3 \mathrm{ml}$ ). Then add the corresponding alkylhalogenide $(0.0044 \mathrm{Mol})$ and reflux at $70^{\circ} \mathrm{C}$ for $30 \mathrm{~min}$. Cool the reaction mixture, filter the precipitate formed, dry and recrystallize from $\mathrm{CH}_{3} \mathrm{CN}$.

\section{Biological Assay}

Antimicrobial and antifungal test

1) Stiff plate agar diffusion method. The study of the antimicrobial and antifungal activity of the compounds synthesized was carried out using the stiff plate agar diffusion method against E. coli, S. aureus, $M$. luteum, $C$. tenuis and $A$. niger [6]. The amount of 
microbial cells was 109 c.f.u./mL. The incubation period for bacteria was $24 \mathrm{~h}$ at $35^{\circ} \mathrm{C}$, for fungi $-48-72 \mathrm{~h}$ at $28-30^{\circ} \mathrm{C}$. Antibiotics vancomicin, oxacillin, nystatin were used as standards. The bacterial cultures, standards and the substances obtained were applied across grooves in the concentration of $5 \mathrm{mg} / \mathrm{mL}$, and then allowed to diffuse in the agar nutrient plate. The antimicrobial effect and the degree of activity of the compounds tested were evaluated by measuring of diameters of the inhibition zones (insensitive: $11-15 \mathrm{~mm}$; sensitive: $16-25 \mathrm{~mm}$; highly sensitive $>25 \mathrm{~mm}$ ). All experiments were repeated three times.

2) Serial dilution method. The minimal inhibitory concentration (MIC) was determined using serial dilutions in the liquid nutrient medium by the classical method [7]. Innoculations were made in the meat infusion broth at $\mathrm{pH} 7.0$ for bacteria or in the wort agar for fungi with different concentrations of the compounds tested. The amount of microbial cells was 109 c.f.u./mL

Results were calculated after storing the control and test tubes for $24-72 \mathrm{~h}$ (at $37^{\circ} \mathrm{C}$ for bacteria and at $30^{\circ} \mathrm{C}$ for fungi) by determining the presence or absence of microbial growth in the medium containing various dilutions of the compound tested. The last tube with growth inhibition (transparent broth) was considered to be the MIC of the compound against the given strain.

In the test tubes $0.02 \mathrm{~mL}$ of the transparent medium was innoculated in the sterilized meat infusion broth (for bacteria) or the wort agar (for fungi) in a stiff plate. The incubation period for bacteria was $24 \mathrm{~h}$ at $35^{\circ} \mathrm{C}$, for fungi $-48-72 \mathrm{~h}$ at $28-30^{\circ} \mathrm{C}$. The minimal bactericidal concentration (MBC) and the minimal fungicidal concentration (MFC) were calculated by the absence of the colony growth. All experiments were repeated three times.

\section{Conclusions}

The aim of the present study was to synthesize and investigate the antibacterial and antifungal activities of some new thienopyrimidine derivatives. The starting material $1\left(\mathrm{R}_{1}, \mathrm{R}_{2}\right.$-thieno[2,3- $\left.d\right]$ pyrimidine$4(3 \mathrm{H})$-one $)$ was used to synthesize the corresponding thiones and selenones. The reaction of alkylation of the compounds obtained was studied. In the case of thieno[2,3- $d]$ pyrimidine- $4(3 H)$-ones the products of $\mathrm{N}$-alkylation were obtained. When thieno[2,3- $d]$ pyrimidine-4(3H)-thio(seleno)nes were alkylated, $\mathrm{S}$ - and Se- derivatives were obtained. Changing of the reaction conditions did not lead to $\mathrm{N}$-alkylated products as it was expected.

The antibacterial and antifungal activities of the compounds obtained in vitro have been screened using the stiff plate agar diffusion method and the serial dilution method. According to the stiff plate agar diffusion method compounds $\mathbf{4 a}$ and $\mathbf{4 c}$ have revealed a significant activity against Escherichia coli, S. aureus, and $M$. luteum appeared to be the most sensitive to compound $10 \mathrm{a}$ in the concentration of $0.5 \%$, it has also been confirmed by the serial dilution method (MIC $1=31.2 \mu \mathrm{g} / \mathrm{mL}$; MBC $=125 \mu \mathrm{g} / \mathrm{mL}$ for M. luteum). The best fungistatic indicators against A.niger have been found for compounds $\mathbf{4 a}$ and $\mathbf{9 a}$ (MIC $=31.2 \mu \mathrm{g} / \mathrm{mL}$ ).

\section{References}

1. 1. Suresh M., Kumar A. S., Gorle S. Letters in Drug Design and Discover, 2013, Vol. 10, No.2. pp.186.

2. Sleebs B. E., Nikolakopoulos G., Street I. P. Bioorg. and Med. Chem. Letters., 2011, Vol. 21, No.19, pp.5992.

3. Khulud M., Al-Taisan, Hassan M., Al-Hazimi A., Shihry S. S. Molecules, 2010, Vol. 15, pp.3932.

4. Romashov L. V., Zeifman A. A., Zakharenko A. I., Novikov F. N., Stroilov V. S. Mendeleev Commun., 2012, Vol. 22, pp.15.

5. Nirogi R. V. S., Kambhampati R. S., Kothmirkar P., Arepalli S., Pamuleti N. R. G., Shinde A. K., Dubey P. K. Synth. Commun., 2011, Vol. 41, pp.2835.

6. Miyashita A., Fujimoto K., Okada T., Higashino T. Heterocycle, 1999, Vol. 42, pp.691.

7. Talukdar P. B., Sengupta S. K., Datta A. K. Indian J. Chem., 1981, Vol. 20, No.7, pp.538-542.

8. Perrissin M., Favre M., Luu-Duc C., Bakri-Logeais F, Narcisse G. Eur. J. Med. Chem., 1984, Vol. 19, No.5, pp.420-424.

Надійшла до редакції 05.11.2015 р. 